\title{
Excitation of wakefield around pulsars
}

\section{Vazha I. Berezhiani}

School of Physics, Free University of Tbilisi, 0183-Tbilisi, Georgian

\section{Zaza Osmanov*}

School of Physics, Free University of Tbilisi, 0183-Tbilisi, Georgia

E-mail: z.osmanovefreeuni.edu.ge

\section{Milivoj Belic}

Science Program, Texas A\&M University at Qatar, PO Box 23874 Doha, Qatar Department of Electronic Engineering

\begin{abstract}
We study the generation of the wakefields by means of the high energy radiation of pulsars. The problem is considered in the framework of a one dimensional approach. We linearize the set of governing equations consisting of the momentum equation, continuity equation and Poisson equation and show that a wavelike structure will inevitably arise relatively close to the pulsar.
\end{abstract}

The Modern Physics of Compact Stars 2015

30 September 2015 - 3 October 2015

Yerevan, Armenia

${ }^{*}$ Speaker. 


\section{Introduction}

It is strongly believed that media around pulsars are efficiently perturbed by their powerful high energy emission, which in turn is directly related to the neutron star's rotation. The total energy of these objects can provide is $W_{\text {tot }}=I \Omega^{2} / 2$, where $I=2 M R_{\star}^{2} / 5$ is pulsar's moment of inertia, $M \approx 1.5 \times M_{\odot}$ and $R_{\star} \approx 10^{6} \mathrm{~cm}$ are pulsar's mass and radius respectively, $M_{\odot} \approx 2 \times 10^{33} \mathrm{~g}$ is the solar mass, $\Omega=2 \pi / P$ is the angular velocity of rotation and $P$ is the corresponding period. It is evident that the rotational energy budget of the pulsar increases for rapidly rotating objects and for millisecond pulsars this might become very large. Indeed, by applying the typical parameters of millisecond pulsars, one can see that this energy is of the order of

$$
W_{\mathrm{tot}} \approx 2.4 \times 10^{52} \times\left(\frac{P}{0.01 s}\right)^{-2} \times\left(\frac{M}{1.5 M_{\odot}}\right) \text { ergs. }
$$

As we see, if even a tiny fraction of $W_{\text {tot }}$ is transformed into radiation, its role might be significant.

It is well known from observations that all pulsars slows down, which in turn means that their released rotational energy can strongly influence their overall emission pattern. The corresponding parameter is called the slow down rate $\dot{P} \equiv d P / d t<0$. It is straightforward to estimate the energy released in unit of time (slow-down luminosity) $L_{\mathrm{sd}} \equiv \dot{W}_{\text {tot }}=I \Omega|\dot{\Omega}|$, where $\dot{\Omega} \equiv d \Omega / d t=-2 \pi \dot{P} / P^{2}$

$$
L_{\mathrm{sd}} \approx 9.5 \times 10^{38} \times\left(\frac{P}{0.01 \mathrm{~s}}\right)^{-3} \times\left(\frac{\dot{P}}{10^{-13} \mathrm{ss}^{-1}}\right) \times\left(\frac{M}{1.5 M_{\odot}}\right) \mathrm{ergs} \mathrm{s}^{-1} .
$$

As we see, even if a tiny fraction of this huge amount of power transforms into that of emission one might have interesting consequences. It is commonly assumed that on average only $1 \%$ of rotational energy is converted to radiation, therefore its luminosity is given by

$$
L \approx 9.5 \times 10^{36} \times\left(\frac{\kappa}{0.01}\right) \times\left(\frac{P}{0.01 \mathrm{~s}}\right)^{-3} \times\left(\frac{\dot{P}}{10^{-13} \mathrm{ss}^{-1}}\right) \times\left(\frac{M}{1.5 M_{\odot}}\right) \mathrm{ergs} \mathrm{s}^{-1} .
$$

where we have taken into account the expression for the bolometric luminosity $L \approx \kappa L_{\mathrm{sd}}$, where $\kappa$ is the fraction of the rotational energy that is converted into radiation. Such a huge radiation power will inevitably undergo the overall pattern around a pulsar and since the radiated energy is very high such a pattern probably might be seen also on distant regions from the central object - thus in the nebular structure.

Apart from the astrophysical example of pulsars discussed above, it is clear that similar physics is encountered in the interactions of strong electromagnetic pulses with plasma in laboratory. In particular, Tajima \& Dawson [1] considered short laser radiation and its effect on plasmas. The authors examined radiation with power density of the order of $10^{18} \mathrm{~W} / \mathrm{cm}^{2}$ irradiating a plasma with particle number densities of the order of $10^{18} \mathrm{~cm}^{-3}[1]$. The group velocity of the electromagnetic radiation is given by

$$
v_{g}^{E M}=c\left(1-\frac{\omega_{p}^{2}}{\omega^{2}}\right)^{1 / 2}<c,
$$

where $\omega_{p} \equiv \sqrt{4 \pi n_{0} e^{2} / m}$ is the plasma frequency, $n_{0}$ is the number density of plasma particles, $e$ and $m$ are respectively the charge and mass of an electron and $\omega$ is the photon frequency. According 
to Ref. [1] the interaction of the radiation with the group velocity (1.4) with plasma creates a wakefield with the phase velocity

$$
v_{p}=\frac{\omega_{p}}{k_{p}}=v_{g}^{E M},
$$

where $k_{p}$ is the wave-number of the plasmon. From Eq. (1.5) it is evident that the Lorentz factor of the plasmon is of the order of $\gamma \approx \omega / \omega_{p}$, which, as we will see later, could be very large in the realistic astrophysical scenarios. As explained in Ref. [1] the electromagnetic waves lead to transverse oscillations of electrons. In the nonrelativistic limit the corresponding average energy of oscillations might be estimated as to be

$$
\left\langle\Delta W_{T}\right\rangle \approx \frac{e^{2}}{2 m \omega^{2}}\left\langle E_{y}^{2}\right\rangle
$$

where $E_{y}$ is the transverse component of the electric field. The momentum the electrons pick up is of the order of [1]

$$
\left\langle\Delta p_{x}\right\rangle \approx \frac{\left\langle\Delta W_{T}\right\rangle}{c} .
$$

During the time duration of the pulse, $\tau$, the electrons are displaced by the lengthscale $\Delta x=\left\langle\Delta v_{x} \tau\right\rangle$. After passing the pulse the charge separation pulls the electrons back and as a result the plasma oscillations are generated. Due to propagation of the excited wakefield the electrons are trapped and accelerated. In the rest frame of plasmons the electrons gain energy, $W \approx e \varphi$, which in the lab frame transforms to [1]

$$
W_{\max } \approx 2 \gamma^{2} \varphi,
$$

where $\varphi$ is the potential in the wake. From Eq. (1.8) it is evident that the wakefield might efficiently accelerate particles. The authors of Ref. [1] have shown that short duration pulses of electromagnetic radiation might efficiently induce the Langmuir waves.

On the other hand, unlike the laboratory processes, in astrophysical situations radiation of astrophysical sources is characterized by a broad band emission. Despite this difference the major mechanism of wakefield generation still works, because during the interaction of very high energy radiation with plasma particles the ions and lighter electrons behave differently. This difference will inevitably lead to charge separation creating an electrostatic field, which in turn will act on the particles. The interaction of high intensity (of the order of $10^{27} \mathrm{ergs} \mathrm{cm}^{-2} \mathrm{~s}^{-1}$ ) radiation with plasmas having density $n$ has been studied by Zampieri et al. [2]. To study the interaction of high energy radiation with the plasma particles the authors considered the following equations

$$
\begin{aligned}
\gamma_{e} \frac{d}{d t}\left(\gamma_{e} m_{e} v_{e}\right) & =-\gamma_{e} Z e E+F_{\mathrm{rad}}, \\
\gamma_{i} \frac{d}{d t}\left(\gamma_{i} m_{i} v_{i}\right) & =\gamma_{i} Z e E,
\end{aligned}
$$

where by $\gamma_{e}$ and $m_{e}$ denote the Lorentz factor and mass of electrons and $\gamma_{i}$ and $m_{i}$ are the same quantities for ions, $v_{e, i}$ are their velocities, $Z$ is the atomic number of ions, $E$ is the induced electric field and

$$
F_{\mathrm{rad}}=-\int \frac{\varepsilon_{1} \mu_{1}-\varepsilon \mu}{c} \gamma_{e}\left(1-\frac{v_{e}}{c} \mu\right) \frac{I_{\varepsilon}}{\varepsilon} \frac{d \sigma}{d \Omega_{s}} d \varepsilon d \Omega d \Omega_{s},
$$


is the radiative force acting on electrons, $I_{\varepsilon}$ is the specific intensity of radiation, $\Omega$ denotes the solid angle subtended by the astrophysical source and $\Omega_{s}$ is the solid angle over the scattering angle, $\varepsilon$ and $\varepsilon_{1}$ are the energies of photons in the laboratory frame (LF) of reference, $\mu\left(\mu_{1}\right)$ are cosines of angles between radial and incident directions respectively and $d \sigma / d \Omega$ is the KleinNishina differential cross section [8]. By applying the Gauss's law, $E=4 \pi Z e n \Delta x$, where $n$ is the number density of charges and $\Delta x=r_{e}-r_{i}$ is the charge separation the authors reduce the system of equations (1.9)-(1.11) to

$$
\begin{gathered}
\frac{d^{2} x_{e}}{d t^{2}}=-\frac{4 \pi Z^{2} e^{2} n}{m_{e}} \Delta x+\frac{\sigma_{T} F}{m_{e} c}, \\
\frac{d^{2} x_{i}}{d t^{2}}=\frac{4 \pi Z^{2} e^{2} n}{m_{i}} \Delta x,
\end{gathered}
$$

where $F \equiv L / 4 \pi r_{e}^{2}$ is the radiation intensity and $\sigma_{T} \approx 6.65 \times 10^{-25} \mathrm{~cm}^{2}$ is the Thomson cross section. By solving and analyzing these equations the authors have argued that radiationinduced electric field might efficiently influence the maximum energy of plasma particles.

The generation of wakefield has been considered in a series of works. Gorbunov \& Kirsanov [3] have examined the nonlinear excitation of longitudinal electrostatic waves in plasmas. These authors studied a set of equations governing the process of wakefield excitation by applying the hydrodynamic equation

$$
\frac{\partial p_{\perp}}{\partial t}+v \frac{\partial p_{\perp}}{\partial x}=e E-\frac{e}{c} v_{\|} B
$$

and two Maxwell's equations

$$
\begin{aligned}
& \frac{\partial E}{\partial x}=-\frac{1}{c} \frac{\partial B}{\partial t}, \\
& \frac{\partial B}{\partial x}=-\frac{1}{c} \frac{\partial E}{\partial t}+\frac{4 \pi e}{c} n v_{\perp},
\end{aligned}
$$

where $v_{\perp}$ and $v_{\|}$are the transverse and longitudinal velocity components of electrons and $p_{\perp}$ is the corresponding transverse component of momentum. Assuming that the transverse velocity is given by

$$
v_{\perp}(x, t)=\frac{1}{2}\left[a(\chi) e^{-i \omega t+i k x}+a^{*}(\chi) e^{i \omega t-i k x}\right],
$$

where the dispersion relation is $\omega^{2}=k^{2} c^{2}+\omega_{p}^{2}$ and $\chi=x-v_{g} t$, where $v_{g}$ is the group velocity of the wave, the authors find from Eqs. (1.14)-(1.17) the following low frequency perturbation

$$
\frac{\delta n_{0}}{n_{0}}=\frac{1}{4 v_{g}^{2}}\left(1-2 \sin ^{2}\left[k_{p}\left(\frac{\chi}{2}-\frac{L}{4}\right)\right]\right),
$$

for $L / 2>\chi>-L / 2$ and

$$
\frac{\delta n_{0}}{n_{0}}=\frac{1}{2 v_{g}^{2}} \sin \left(k_{p} \chi\right) \sin \left(\frac{k_{p} L}{2}\right),
$$

for $\chi<-L / 2$, where $k_{p}=\omega_{p} / v_{g}$. On the basis of these results Gorbunov \& Kirsanov [3] showed that the wakefield excited behind the packet can strongly affect particle acceleration. They argued that particles injected into the plasma are accelerated quite efficiently. The change in the sign of 
electric field leads to a deceleration phase. The overall effect is that the electrons trapped in the wakefield region have maximal energy given by

$$
\varepsilon_{\text {max }}=\varepsilon_{0} \gamma \frac{e E}{m_{e} \omega_{p} c}\left(1+\left[1+\frac{2 m_{e} k_{p}}{e E \gamma}\right]^{1 / 2}\right),
$$

where $\varepsilon_{0}$ is the initial energy of electrons, $e$ is the elementary charge.

In Ref. [4] the authors have examined the case of relativistic intense pulse. To study the role of linearly polarized electromagnetic waves on the ambient plasma these authors considered the equation governing the evolution of a dimensionless vector potential, $A \equiv p_{\perp} / m c$, describing the pulse $A=0.5\left[a(\chi, t) \exp \left(-e \omega_{0} t+i k_{0} x\right)+c . c.\right]$, where $k_{0}$ is the wave vector of radiation and $\omega_{0}^{2}=k_{o}^{2} c^{2}-\omega_{p}^{2}, \chi=x-v_{g} t, v_{g}=c^{2} k_{0} / \omega_{0}$. This equation, given by Ref. [4], reads

$$
\begin{aligned}
& 2 i \omega_{0} \frac{\partial a}{\partial t}+\frac{\omega_{p}^{2}}{\omega_{0}^{2}} c^{2} \frac{\partial^{2} a}{\partial \chi^{2}}+2 v_{g} \frac{\partial a}{\partial t \partial \chi}=-\omega_{p}^{2} \frac{\psi_{0}}{1+\psi_{0}} a, \\
& \frac{d^{2} \psi_{0}}{d \chi^{2}}-k_{p}^{2} \frac{1+|a|^{2} / 2-\left(1+\psi_{0}\right)^{2}}{2\left(1+\psi_{0}\right)^{2}}=0,
\end{aligned}
$$

where, as above, $k_{p}=\omega_{p} / v_{g}$ and $\psi_{0}$ is the low frequency component of the potential. A laser pulse with a wavelength of the order of $10 \mu \mathrm{m}$ and intensity $10^{17} \mathrm{~W} / \mathrm{cm}^{2}$ having an edge length of $0.1 \mathrm{ps}$ was considered. Using Eqs. (1.21) and (1.22) it was shown that the pulse will accelerate the electrons up to eneregies $1 \mathrm{GeV}$ over a distance $50 \mathrm{~cm}$ in a plasma medium with number density of the order of $10^{15} \mathrm{~cm}^{-3}$.

The problem of generation of wakefield has been examined by Berezhiani \& Murusidze in Ref. [5] . To address this problem the authors used the Maxwell's equations in combination with the equations of motion and derived the following equation for electron momentum

$$
\frac{\partial^{2} p}{\partial t^{2}}-\Delta p+\nabla(\nabla \cdot p)+\frac{\partial}{\partial t} \nabla \sqrt{1+p^{2}}+\frac{p}{\sqrt{1+p^{2}}}\left[1+\partial(\nabla \cdot p) / \partial t+\Delta \sqrt{1+p^{2}}\right]=0,
$$

which for the transparent plasma medium reduces to

$$
\frac{d^{2} y}{d x^{2}}=\frac{1}{2}\left(\frac{\gamma_{\perp}^{2}}{y^{2}}-1\right)
$$

where $\gamma_{\perp}^{2}=1+p_{\perp}, x=k_{p}(z-c t), y=\sqrt{\gamma_{\perp}^{2}+p_{\|}^{2}}-p_{\|}=1+\Phi$ and $\Phi \approx e \Phi / m c^{2}$ is the dimensionless potential. By analyzing Eq. (1.24) it was shown that the maximum increase of energy of trapped electrons is given by

$$
\Delta E=2 \gamma_{g}^{2} m c^{2} \Delta \Phi_{\max },
$$

where $\gamma_{g}=\left(1-v_{g}^{2} / c^{2}\right)^{-1 / 2}$. Therefore, Berezhiani \& Murusidze [5] have shown that longitudinal oscillations with relativistic phase velocities lead to the excitation of the wakefield.

Finally, we note that Sprangle et al. [6] developed a nonlinear theory of laser-plasma interactions and studied the relativistic optical guiding, nonlinear excitation of wakefields and generation of coherent harmonic radiation. 
In this paper we consider the possibility of excitation of wakefields via the interaction of the pulsar high energy emission with plasma surrounding the pulsar. Generally speaking, pulsars are emitting in narrow channels, which in turn are rotating. Therefore any irradiated area surrounding the pulsar experiences the radiation pressure periodically, which can lead to generation of wakefields.

The structure of the paper is as follows. In Sec. II we develop an analytical method for studying the generation of wakefields in media, periodically irradiated by the pulsar's high-energy emission. In Sec. III we present our results and summarize them in the Sec. IV.

\section{Theoretical background}

In this section we consider the set of equations which govern the generation of wakefields. In the framework of our model this structure is formed due to the high-energy radiation pressure of a pulsar. On the other hand this pressure is created by means of the Compton interaction of radiation photons and electrons in the medium surrounding the neutron star. The corresponding energy of scattered electrons has the following form (see Ref. [7])

$$
\varepsilon_{1}^{\prime}=\frac{\varepsilon^{\prime}}{1+\left(\varepsilon^{\prime} / m c^{2}\right)\left(1-\cos \chi^{\prime}\right)},
$$

where $\varepsilon^{\prime}$ and $\varepsilon_{1}^{\prime}$ are the photon energies before and after scattering respectively in the rest frame of the electron, $m$ is its mass, $c$ is the speed of light and $\chi^{\prime}$ is the angle between the incident and scattered photon directions.

In the framework of the Thomson scattering, $\varepsilon_{1}^{\prime} \approx \varepsilon^{\prime}$, Eq. (1.11) reduces to [2]

$$
F_{r a d}=\sigma_{T} \frac{F}{c}
$$

where $F$ is given by

$$
F=\int I_{\varepsilon} \mu d \varepsilon d \Omega=\frac{L}{4 \pi r^{2}(1-\cos [\theta / 2])},
$$

where it has been taken into account that each radiation channel carries half of the luminosity. The corresponding solid angle of the cone is $2 \pi(1-\cos [\theta / 2])$ and $\theta$ is the opening angle of the radiation cone.

The system of equations governing the generation of wakefields consists of the momentum equation [3]

the continuity equation

$$
\frac{\partial v_{\|}}{\partial t}+v_{\|} \frac{\partial v_{\|}}{\partial x}=-\frac{e}{m} \frac{\partial \varphi}{\partial x}+f_{\mathrm{rad}}
$$

$$
\frac{\partial n}{\partial t}+\frac{\partial}{\partial x}\left(n v_{\|}\right)=0
$$

and the Poisson equation

$$
\frac{\partial^{2} \varphi}{\partial x^{2}}=-4 \pi e\left(n-n_{0}\right)
$$

where $v_{\|}$is the velocity component parallel to the $x$-axis, $\varphi$ denotes the charge separation potential, $f_{\text {rad }}=F_{\text {rad }} / m, n$ is the electron's number density and $n_{0}$ is the unperturbed number density. 
We solve the problem of generation of the wakefield by linearizing the governing equations, which might be easily reduced to

$$
\frac{\partial^{2} \delta n}{\partial t^{2}}+\omega_{p}^{2} \delta n=-n_{0} \frac{\partial f_{\mathrm{rad}}}{\partial x},
$$

where by $\delta n$ we denote the density perturbation.

Since the excited modes are traveling waves it is convenient to introduce the variable $\xi \equiv$ $x-c t$, which leads to the following form of the poisson equation

$$
\frac{\partial^{2} \varphi}{\partial \xi^{2}}=-4 \pi e \delta n
$$

By applying this expression to Eq. (2.7) one gets

$$
\frac{\partial^{4} \varphi}{\partial \xi^{4}}+\frac{\omega_{p}^{2}}{c^{2}} \frac{\partial^{2} \varphi}{\partial \xi^{2}}=\frac{m \omega_{p}^{2}}{e c^{2}} \frac{\partial f_{\mathrm{rad}}}{\partial \xi},
$$

which straightforwardly can be reduced to

$$
\frac{\partial^{2} \varphi}{\partial \xi^{2}}+\frac{\omega_{p}^{2}}{c^{2}} \varphi=\frac{m \omega_{p}^{2}}{e c^{2}} \int f_{\mathrm{rad}}(\xi) d \xi
$$

By assuming that $f_{\text {rad }}(\xi)$ is a step-like function, the solution of Eq. (2.10) can be expressed as follows

$$
\varphi(\beta)=\frac{m f_{\mathrm{rad}, 0} \xi_{0}}{e}\left[1-\frac{\sin \alpha}{\alpha} \cos (\alpha\{\beta-1\})-\frac{1-\cos \alpha}{\alpha} \sin (\alpha\{\beta-1\})\right],
$$

where $f_{\text {rad }, 0}$ is the radiation reaction force, $\xi_{0}=c \theta / \Omega, \alpha=\omega_{p} \xi_{0} / c$ and $\beta=\xi / \xi_{0}$.

\section{Discussion}

We now examine generation of wakefields inside the nebula around a pulsar. For this purpose we consider the Crab nebula parameters: mass $M_{N b} \sim 4 M_{\odot}$ (see Ref. [9]) and radius $R_{N b} \sim 1.7 \mathrm{pc}$, which thus define the average number density of matter $n_{N b} \sim 3 M_{N b} / 4 \pi R_{N b}^{3} \sim 8 \mathrm{~cm}^{-3}$. We will assume below that escaping particles do not change the average density of the Crab nebula. The Goldreich-Julian number density is given by [10]

$$
n_{G J}=\frac{\Omega B_{s t}}{2 \pi e c} \times\left(\frac{R_{\star}}{r}\right)^{3},
$$

where $B_{\star} \approx 1.8 \times 10^{12} \times \sqrt{P \dot{P}} G$ is the surface magnetic field of the pulsar. On the other hand, outside the rotationally driven region (magnetosphere) the behaviour of magnetic field is $1 / r$ (see Ref. [11]), which leads to the following condition

$$
r \gg 1.2 \times 10^{-6} \times\left(\frac{P}{0.01 s}\right)^{-5 / 2} \times\left(\frac{\dot{P}}{10^{-13} s s^{-1}}\right)^{1 / 2} \times\left(\frac{R_{\star}}{10^{6} \mathrm{~cm}}\right)^{3} \times\left(\frac{n_{N b}}{10 \mathrm{~cm}^{-3}}\right)^{-1} \mathrm{AU}
$$


It is clear that the effect we are interested in strongly depends on the luminosity of a pulsar. In general there is a special class of pulsars, the so called $X$-ray pulsars, which exhibit extremely high values of luminosity. It is believed that their emission has a synchrotron origin. This process is maintained due to the quasi-linear diffusion (see Ref. [12]), when the opening angle of a radiation cone is expressed as [13]

$$
\theta \approx 0.14 \times\left(\frac{0.01 s}{P}\right)^{1 / 2} \times\left(\frac{R_{\star}}{10^{6} \mathrm{~cm}}\right)^{1 / 2} \mathrm{rad} .
$$

If the electrons are trapped inside the wakefield, they will inevitably experience the potential difference [see Eqs. (2.2),(2.3),(1.3) and (3.3)]

$$
\begin{aligned}
\varepsilon(\mathrm{eV}) & \approx 5.4 \times\left(\frac{\kappa}{0.01}\right) \times\left(\frac{P}{0.01 \mathrm{~s}}\right)^{-5 / 2} \times\left(\frac{\dot{P}}{10^{-13} \mathrm{ss}^{-1}}\right) \\
& \times\left(\frac{M}{1.5 M_{\odot}}\right) \times\left(\frac{R_{\star}}{10^{6} \mathrm{~cm}}\right)^{-1 / 2} \times\left(\frac{r}{0.01 \mathrm{AU}}\right)^{-2} .
\end{aligned}
$$

It is seen that, for example, on distances $0.01 \mathrm{AU}$ from the Crab pulsar the potential difference will be of the order of $4 \mathrm{eV}$; for $r \sim 0.001 \mathrm{AU}$ we find $\varepsilon(\mathrm{eV}) \sim 0.4 \mathrm{keV}$.

Since the most energetic pulsars are the so called newly born young millisecond pulsars [14], it is interesting to estimate the wakefield generation for this particular class of pulsars too. By considering $P \sim 0.001$ s and $\dot{P} \sim 10^{-12} \mathrm{ss}^{-1}$ one can show that for 0.01 AU the potential difference will be $\sim 2 \mathrm{keV}$.

\section{Summary}

1. We have examined the excitation of wakefield in the media surrounding a pulsar. The problem has been studied in a one dimensional approximation, assuming that the particles follow along the magnetic field lines. Therefore, we started from the one-dimensional form of the momentum equation, the continuity equation and the Poisson equation, linearized them, and solved the resulting equations in the case where the radiation force has a simple step-like form.

2. The model has been applied to the Crab-like pulsars and the newly born young millisecond pulsars. It was shown that in case of the Crab-like pulsars, trapped electrons will be subject to the potential difference of the order of $0.4 \mathrm{keV}$, whereas for the newly born pulsars this value can be even higher, of the order $2 \mathrm{keV}$.

\section{Acknowledgments}

ZO was partially supported by the Shota Rustaveli National Science Foundation grant (N31/49).

\section{References}

[1] Tajima, T., \& Dawson, J. M., 1979, Phys. Rev. Lett., 43, 267 
[2] Zampieri, L., Turolla, R., Foschini, L. \& Treves, A., 2003, ApJ, 592, 368

[3] Gorbunov, L. M. \& Kirsanov, V. I., 1987, Sov. J. Exp. Theor. Phys, 93, 509

[4] Bulanov, S. V., Kirsanov, V. I. \& Sakharov, A. S., 1987, Sov. J. Exp. Theor. Phys., 50, 176

[5] Berezhiani, V. I. \& Murusidze, I. G., 1990, PhLA, 3148, 338

[6] Sprangle, P., Esarey, E. \& Ting, A., 1990, PhRvL, 64, 2011

[7] Rybicki, G.B. \& Lightman, A. P., 1979, Radiative Processes in Astrophysics. Wiley, New York

[8] Blumenthal, G. R., \& Gould, R. J., 1970, Rev. Mod. Phys., 42, 237

[9] Hester, J.J., 2008, ARA\&A, 46, 127

[10] Goldreich, P. \& Julian, W.H., 1996, ApJ, 157, 869

[11] Manchester, R.N. \& Taylor, J.H., 1980, Pulsars, F.H. Freeman and Company

[12] Machabeli, G. \& Osmanov, Z., 2009, ApJL, 700, 114

[13] Machabeli, G. Z. \& Usov, V. V., 1979, Sov. Astr. L, 5, 238

[14] Carroll, B.W. \& Ostlie, D.A. An Introduction to Modern Astrophysics, Pearson International Edition (2006) 